\title{
Application of Blood-Brain Barrier Permeability Imaging in Global Cerebral Edema
}

\author{
(D). Ivanidze, (D).N. Kallas, (D)A. Gupta, (DE. Weidman, (D). Baradaran, (DD. Mir, (D)A. Giambrone, (D)A.Z. Segal, (D). Claassen, and \\ (DP.C. Sanelli
}

\begin{abstract}
BACKGROUND AND PURPOSE: Blood-brain barrier permeability is not routinely evaluated in the clinical setting. Global cerebral edema occurs after SAH and is associated with BBB disruption. Detection of global cerebral edema using current imaging techniques is challenging. Our purpose was to apply blood-brain barrier permeability imaging in patients with global cerebral edema by using extended CT perfusion.
\end{abstract}

MATERIALS AND METHODS: Patients with SAH underwent CTP in the early phase after aneurysmal rupture (days $0-3$ ) and were classified as having global cerebral edema or nonglobal cerebral edema using established noncontrast CT criteria. CTP data were postprocessed into blood-brain barrier permeability quantitative maps of PS (permeability surface-area product), $K^{\text {trans }}$ (volume transfer constant from blood plasma to extravascular extracellular space), $\mathrm{K}_{\mathrm{ep}}$ (washout rate constant of the contrast agent from extravascular extracellular space to intravascular space), VE (extravascular extracellular space volume per unit of tissue volume), VP (plasmatic volume per unit of tissue volume), and $\mathrm{F}$ (plasma flow) by using Olea Sphere software. Mean values were compared using $t$ tests.

RESULTS: Twenty-two patients were included in the analysis. $K_{\text {ep }}(1.32$ versus $1.52, P<.0001), K^{\text {trans }}(0.15$ versus $0.19, P<.0001)$, VP $(0.51$ versus $0.57, P=.0007)$, and $\mathrm{F}$ (1176 versus $1329, P=.0001)$ were decreased in global cerebral edema compared with nonglobal cerebral edema while VE $(0.81$ versus $0.39, P<.0001)$ was increased.

CONCLUSIONS: Extended CTP was used to evaluate blood-brain barrier permeability in patients with SAH with and without global cerebral edema. $\mathrm{K}_{\mathrm{ep}}$ is an important indicator of altered blood-brain barrier permeability in patients with decreased blood flow, as $\mathrm{K}_{\mathrm{ep}}$ is flow-independent. Further study of blood-brain barrier permeability is needed to improve diagnosis and monitoring of global cerebral edema.

ABBREVIATIONS: BBBP = blood-brain barrier permeability; EES = extravascular extracellular space; $\mathrm{F}=$ plasma flow; GCE = global cerebral edema; IVS = intravascular space; $\mathrm{K}_{\mathrm{ep}}=$ washout rate constant of the contrast agent from EES to IVS; $K^{\text {trans }}=$ volume transfer constant from blood plasma to EES; PS $=$ permeability surface-area product; VE = extravascular extracellular space volume per unit of tissue volume; VP = plasmatic volume per unit of tissue volume

$\mathbf{T}$ he blood-brain barrier plays an important role in many CNS diseases relating to microvascular dysfunction and neuroinflammation, which are known to increase blood-brain barrier permeability (BBBP) in animal models. ${ }^{1}$ Obtaining quantitative

Received June 22, 2015; accepted after revision February 22, 2016

From the Departments of Radiology (J.I., O.N.K., A.Gupta, E.W., H.B., D.M., P.C.S.), Healthcare Policy and Research (A.Giambrone), and Neurology (A.Z.S.), Weill Cornell Medical College, New York-Presbyterian Hospital, New York, New York; and Department of Neurology (J.C.), New York-Presbyterian Hospital, Columbia University Medical Center, New York, New York.

This work was supported by the Radiological Society of North America Research and Education Foundation Resident Research Grant (awarded to J.I.), the Brain Aneurysm Foundation Grant (awarded to P.C.S.), and grant 5K23NS058387 from the National Institute of Neurological Disorders and Stroke (NINDS), a component of the National Institutes of Health $(\mathrm{NIH})$. Its contents are solely the responsibility of the authors and do not necessarily represent the official view of NINDS or NIH.

Paper previously presented at: Annual Meeting of the American Society of Neuroradiology, April 25-30, 2015; Chicago, Illinois.
BBBP information in the clinical setting would constitute a novel diagnostic and therapeutic strategy with widespread application. Global cerebral edema (GCE) represents a major complication in patients with $\mathrm{SAH}$, is an independent predictor of SAH morbidity and mortality, ${ }^{2,3}$ and is thought to be related to disruption of the BBB. Moreover, delayed cerebral ischemia represents an independent major complication and poor prognostic factor in patients with SAH. ${ }^{4}$ Initial studies have shown that earlier diagnosis of delayed cerebral ischemia before manifestation of clinical symptoms, altered $\mathrm{CBF}$, and NCCT findings is possible with BBBP imaging by using CTP. ${ }^{5} \mathrm{BBBP}$ evaluation with $\mathrm{CTP}$ is a promising

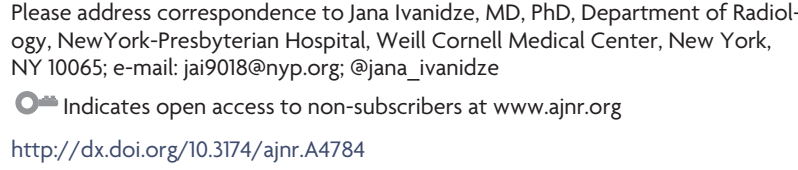

AJNR Am J Neuroradiol 37:1599-603 Sep 2016 www.ajnr.org 

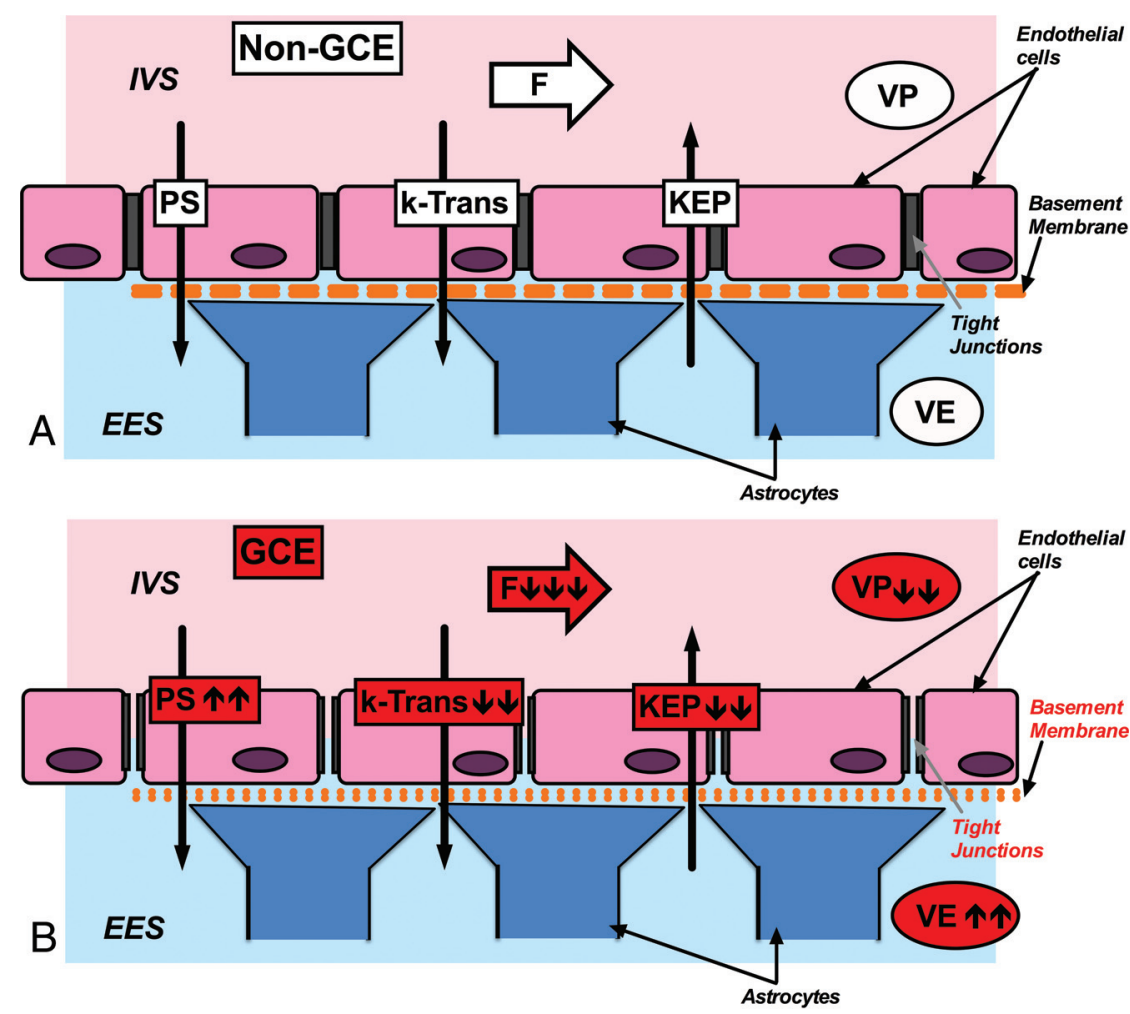

FIG 1. Cytoarchitecture of the BBB and schematic representation of BBBP parameters derived from extended CTP. A, Non-GCE. At baseline, the BBB is composed of endothelial cells connected by tight junctions (as opposed to fenestrated endothelium outside of the CNS), the basement membrane, and the astrocyte processes. $B$, In the setting of GCE, there is an increase in EES as reflected by increased VE and decreased $\mathrm{K}_{\text {ep }}$, indicating increased BBBP (indicated in the drawing by a more porous basement membrane and tight junctions). PS is also elevated, indicating increased BBBP. Importantly, $\mathrm{K}_{\text {ep }}$ is independent of F. In GCE, $\mathrm{K}_{\text {ep }}$ is the most reliable parameter to evaluate BBBP in the setting of flow impairment.

technique, especially in patients with $\mathrm{SAH}$, given that these patients are critically ill, require intensive monitoring, and often have contraindications to evaluation with MR imaging. ${ }^{5}$ Currently, imaging diagnosis of GCE relies on NCCT demonstrating diffuse effacement of the hemispheric sulci and diffuse obscuration of the hemispheric gray-white matter junction at the level of the centrum semiovale. ${ }^{3}$ These findings are challenging to identify in the setting of diffuse $\mathrm{SAH}$ and increased intracranial pressure effacing the sulci and cisterns. Moreover, NCCT fails to provide a direct assessment of the hemodynamic and microvascular dysfunction thought to be central to the underlying pathophysiology of GCE. Quantitative imaging of microvascular parameters, such as measures of BBBP, may provide for more accurate detection and monitoring of GCE compared with NCCT and could potentially improve the clinical management and outcomes of patients after SAH.

Extended CTP allows measurement of BBBP parameters not currently used in clinical practice. $K_{\mathrm{ep}}$ represents the washout rate constant of the contrast agent from the extravascular extracellular space (EES) to the intravascular space (IVS). $\mathrm{K}_{\mathrm{ep}}$ therefore reflects the flow across the blood vessel wall from the EES to the IVS, and is independent of blood plasma flow. Based on transport equations proposed by Gowland et $\mathrm{al}^{6}$ :

$$
T=1 / K_{\mathrm{ep}},
$$

where $T$ is the time constant for transfer across the BBB. Therefore, $K_{e p}$ is thought to be related inversely to BBBP. $K^{\text {trans }}$ represents the volume transfer constant from the blood plasma to the EES. PS is the permeability surface-area product and represents the flow across the blood vessel wall from the IVS to the EES. VE represents the volume in the EES per unit of tissue volume, and VP represents the plasmatic volume per unit of tissue volume. F represents the plasmatic flow. In summary, $\mathrm{K}_{\mathrm{ep}}$, PS, and $K^{\text {trans }}$ can be viewed as the primary BBBP parameters because they reflect flow across the BBB. ${ }^{6,7}$ On the other hand, VE, VP, and F are descriptive of hemodynamic conditions on either side of the BBB (the IVS or the EES, respectively). Fig 1 summarizes the relationships of BBBP parameters under normal conditions and in the setting of GCE. CTP has numerous practical advantages that make it highly suitable for critically ill patients: it is widely available, can be performed with most scanners, has few contraindications, and has rapid acquisition and processing times. Assessment of these parameters offers a promising technique to evaluate BBBP in the clinical setting. However, it has not been widely adopted in clinical practice, with few published investigations studying measures of BBBP. ${ }^{5,8,9}$

The purpose of our study was to apply BBBP imaging by using extended CTP in the setting of GCE and to quantitatively assess its parameters. We hypothesize that application of extended CTP is able to detect a significant difference in the BBBP parameters in patients with GCE compared with patients without GCE in the early phase after $\mathrm{SAH}$.

\section{MATERIALS AND METHODS \\ Population}

This study was an institutional review board-approved retrospective analysis performed on consecutive patients with SAH enrolled in a prospective diagnostic accuracy trial at our institution. SAH was determined by a combination of NCCT, CTA, DSA, and/or CSF analysis. In this prospective study design, CTP was performed on days $0-3$ after aneurysmal rupture to assess baseline cerebral perfusion and to compare with later examinations for interval change. All patients underwent either endovascular coiling or surgical clipping before CTP. Demographic and clinical data were collected (Table 1).

All patients with SAH with CTP performed on days $0-3$ were included in our study. Exclusion criteria were CTP examinations with severe motion degradation limiting postprocessing methods and unavailable CTP data from archives for postprocessing.

Patients were classified into 3 outcome groups: GCE $(n=11)$, 
Table 1: Clinical characteristics of the study population

\begin{tabular}{lccc}
\hline & $\begin{array}{c}\text { GCE } \\
(\boldsymbol{n}=11)\end{array}$ & $\begin{array}{c}\text { Non-GCE } \\
(\boldsymbol{n}=11)\end{array}$ & $\begin{array}{c}\boldsymbol{P} \\
\text { Value }\end{array}$ \\
\hline Mean age (years) & 51 & 49 & .67 \\
Sex & & & .92 \\
$\quad$ Female & $82 \%(9 / 11)$ & $64 \%(7 / 11)$ & \\
$\quad$ Male & $18 \%(2 / 11)$ & $36 \%(4 / 11)$ & \\
Focal deficit at presentation & $46 \%(5 / 11)$ & $36 \%(4 / 11)$ & .66 \\
Loss of consciousness & $18 \%(2 / 11)$ & $27 \%(3 / 11)$ & .61 \\
Mean Hunt and Hess score & 3.0 & 2.5 & .15 \\
$\quad$ Ventriculostomy catheter & $82 \%(9 / 11)$ & $82 \%(9 / 11)$ & 1.00 \\
Aneurysm location & & & .66 \\
$\quad$ Anterior & $64 \%(7 / 11)$ & $54 \%(6 / 11)$ & \\
$\quad$ Posterior & $36 \%(4 / 11)$ & $46 \%(5 / 11)$ & \\
Treatment $\quad$ & & .19 \\
$\quad$ Coiled & & & \\
$\quad$ Clipped & $27 \%(3 / 11)$ & $54 \%(6 / 11)$ & \\
\hline
\end{tabular}

non-GCE $(n=11)$, and indeterminate $(n=2)$ based on admission NCCT. GCE was defined using the following established criteria $^{3}$ : complete or near-complete effacement of the hemispheric sulci and basal cisterns and bilateral and extensive disruption of the hemispheric gray-white matter junction (including basal ganglia) at the level of the centrum semiovale. ${ }^{3}$ Absence of both features resulted in classification as non-GCE. If only 1 feature was present, patients were classified as indeterminate. Classification was performed independently by 2 board-certified neuroradiologists (15 years and 3 years of experience) blinded to all other clinical and imaging data. Consensus reading was provided for adjudication. Interobserver agreement was analyzed using the $\kappa$ coefficient.

\section{Image Acquisition, Postprocessing, and Quantitative Analysis}

CTP was performed in the early phase (days $0-3$ ) after $\mathrm{SAH}$, along with NCCT and CTA, as part of our study trial protocol. NCCT was performed from the foramen magnum to the vertex by using the following parameters: $120 \mathrm{kVp}, 250 \mathrm{mAs}, 1.0$ rotation time, and 5.0-mm collimation.

Extended CTP scanning was performed on a 64-section scanner (LightSpeed Discovery HD-750; GE Healthcare, Milwaukee, Wisconsin) with an axial shuttle mode protocol for simultaneous acquisition of CTA and CTP data using the following parameters: $80 \mathrm{kVp}, 400 \mathrm{mAs}, 0.4$ rotation time, 5.0-mm collimation with 17 cine cycles, and 2.8-second interscan delay for the first pass. Second pass technique included 10 cine cycles with a 10-second interscan delay. A total of $90 \mathrm{~mL}$ of nonionic contrast was intravenously administered at $4.0 \mathrm{~mL} / \mathrm{s}$ followed by a $30 \mathrm{~mL}$ saline-bolus chaser. Average CT dose index volume was $300 \mathrm{mGy}$ and average dose-length product was $2500 \mathrm{mGy}-\mathrm{cm}$ for the entire examination, including NCCT, CTA, and extended CTP.

For this study, CTP data were postprocessed off-line into maps of the BBBP parameters $\left(K_{e p}, K^{\text {trans }}, P S, F, E\right.$ [extraction fraction of contrast agent], VE, and VP), CBF, CBV, and MTT by using commercially available software (Olea Sphere V.2.0; Olea Medical, La Ciotat, France), based on previously published recommendations and guidelines. ${ }^{8,10}$ Quantitative analysis of CTP data was performed using standardized sampling of the cerebral cortex according to established methods ${ }^{4,11}$ with round, continuous ROIs placed along the cerebral cortex. Up to 24 ROIs were included in each CTP section, distributed in the following territories: approximately 4 in the anterior cerebral artery, 12 in the middle cerebral artery, and 4 in the posterior cerebral artery territories. ROIs overlying large cortical vessels with CBF greater than $100 \mathrm{~mL} / 100$ $\mathrm{g} / \mathrm{min}$ were excluded from the statistical analysis. ${ }^{12}$ Mean values and $95 \%$ CIs were calculated using the remaining ROIs in all CTP sections for each patient. Unpaired $t$ tests were performed to compare $\mathrm{K}_{\mathrm{ep}}, K^{\text {trans }}, \mathrm{PS}, \mathrm{F}$, extraction fraction of contrast agent, VE and VP, as well as CBF, CBV, and MTT in the GCE and non-GCE groups.

\section{Permeability Modeling}

Permeability parameters were calculated using the Delayed Lawrence and Lee Model. ${ }^{13}$ According to the Delayed Lawrence and Lee Model, $\mathrm{K}_{\mathrm{ep}}$ represents the washout rate constant of the contrast agent from the EES to the IVS. The formula for $\mathrm{K}_{\mathrm{ep}}$ is:

$$
K_{\mathrm{ep}}=K^{\mathrm{trans}} / V E \text {. }
$$

$\mathrm{K}_{\mathrm{ep}}$ appears to have an inverse relationship with BBBP, and therefore decreases with increased permeability. Importantly, $K_{e p}$ is independent of flow. ${ }^{6}$

$K^{\text {trans }}$ equals the blood plasma flow per unit volume of tissue, derived through the formula:

$$
K^{\text {trans }}=E \times F
$$

where $E$ represents the extraction fraction of contrast agent that leaves the IVS in the first pass of the bolus through the vascular bed. $E$ has no units assigned and has a value between 0 and $1 . F$ represents the plasma flow per unit tissue volume and its units are $\mathrm{mL} \times \mathrm{mL}^{-1} \times \min ^{-1} . K^{\text {trans }}$ is dependent on the blood flow.

PS represents the flow across the blood vessel wall from the IVS to the EES. The formula for PS is:

$$
P S=-F \times(1-H c t) \times \log (1-E),
$$

where Hct is the hematocrit. According to recommendations contained in the Olea Manual, a default hematocrit level is set to 0.45 for the calculation of all derived perfusion and permeability parameters for which a hematocrit level is required. PS increases with increased permeability; however, PS is also flow-dependent. ${ }^{6}$

In summary, $\mathrm{K}_{\mathrm{ep}}$, PS, and $K^{\text {trans }}$ have previously been used to assess BBBP in patients ${ }^{9,14}$ and animal models. ${ }^{15,16}$ However, according to the Lawrence and Lee Model derivations above, decreasing $\mathrm{K}_{\mathrm{ep}}$ most accurately reflects increasing disruption and permeability of $\mathrm{BBB}$, especially in the setting of impaired CBF, such as occurs in GCE. ${ }^{12}$

\section{RESULTS}

\section{Study Population}

Twenty-two patients were included in the study after excluding indeterminate patients $(n=2)$ from the statistical analysis. In the 22 patients, 50\% (11/22) were classified as having GCE and 50\% $(11 / 22)$ as non-GCE. There was statistically significant high interobserver reproducibility, with $\kappa$ interobserver agreement coefficient of $0.86(P<.0001)$ for sulcal effacement and $0.73(P<$ $.0001)$ for gray-white matter differentiation between the 2 blinded 
Table 2: Quantitative BBBP and CTP results ${ }^{\mathrm{a}}$

\begin{tabular}{lccc}
\hline & GCE & Non-GCE & $P$ Value \\
\hline $\mathrm{K}_{\mathrm{ep}}\left(\mathrm{min}^{-1}\right)$ & $1.28(1.23-1.33)$ & $1.52(1.45-1.59)$ & $<.0001$ \\
$K^{\text {trans }}\left(\mathrm{min}^{-1}\right)$ & $0.15(0.14-0.16)$ & $0.19(0.18-0.20)$ & $<.0001$ \\
$\mathrm{PS}\left(\mathrm{mL} \times \mathrm{mL}^{-1} \times \mathrm{min}^{-1}\right)$ & $227.4(213.4-241.5)$ & $208.4(189.7-227.2)$ & .1095 \\
$\mathrm{VE}$ & $0.70(0.61-0.78)$ & $0.39(0.36-0.42)$ & $<.0001$ \\
$\mathrm{VP}$ & $0.51(0.48-0.53)$ & $0.57(0.54-0.60)$ & .0019 \\
$\mathrm{~F}\left(\mathrm{~mL} \times \mathrm{mL}^{-1} \times \mathrm{min}^{-1}\right)$ & $1175(1125-1226)$ & $1329(1259-1398)$ & .0005 \\
$\mathrm{CBF}\left(\mathrm{mL} \times 100 \mathrm{~g}^{-1} \times \mathrm{min}^{-1}\right)$ & $32.57(28.20-36.93)$ & $42.02(35.44-48.60)$ & .0148 \\
$\mathrm{CBV}\left(\mathrm{mL} \times 100 \mathrm{~g}^{-1}\right)$ & $5.86(4.76-6.96)$ & $5.86(4.16-7.56)$ & .9950 \\
$\mathrm{MTT}(\mathrm{s})$ & $2.28(1.44-3.12)$ & $2.20(1.99-2.40)$ & .8376 \\
\hline
\end{tabular}

${ }^{a}$ Data presented as mean $(95 \% \mathrm{Cl})$.

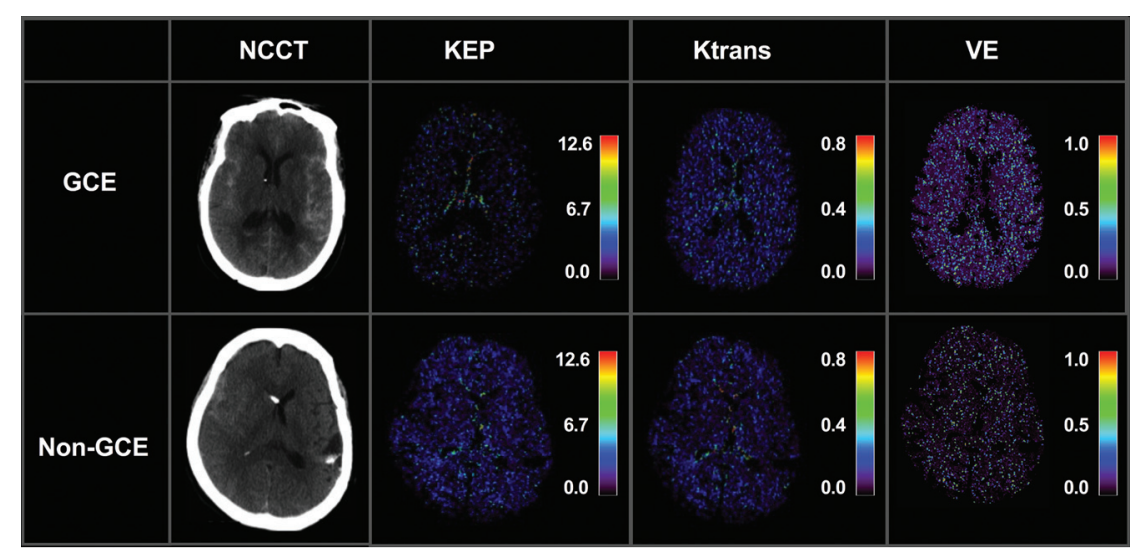

FIG 2. BBBP quantitative maps of $K_{\text {ep }}, K^{\text {trans }}$, and VE in a representative patient from the GCE group (top panel) and a representative patient from the non-GCE group (bottom panel). NCCT images demonstrate loss of gray-white matter differentiation and presence of sulcal effacement in the patient with GCE. Corresponding BBBP maps reveal decreased $\mathrm{K}_{\text {ep }}$, slightly decreased $K^{\text {trans }}$, and increased VE in the same patient compared with the patient without GCE.

neuroradiologists. Clinical characteristics of the study population are summarized in Table 1.

\section{Global BBBP Analysis}

Quantitative BBBP results along with CTP results are summarized in Table $2 . \mathrm{K}_{\mathrm{ep}}, K^{\mathrm{trans}}, \mathrm{VP}$, and $\mathrm{F}$ were significantly decreased, while VE was significantly increased, in the GCE group compared with the non-GCE group. Differences in PS and extraction fraction of contrast agent did not reach statistical significance. However, there was a trend for increased PS in GCE. CBF was significantly decreased in the GCE group compared with the non-GCE group, while differences in CBV and MTT did not reach statistical significance.

Fig 2 demonstrates quantitative BBBP maps in a representative patient from the GCE group and a representative patient from the non-GCE group, respectively.

\section{DISCUSSION}

Assessment of BBBP with CTP is a novel technique that has not been adopted in clinical practice despite its numerous practical advantages, including widespread availability and relatively low cost. Quantitative BBBP evaluation in the clinical setting promises new insights into the underlying pathophysiology of many neurologic diseases. The relationship between BBBP and microvascular dysfunction and neuroinflammation plays an important role in numerous conditions, such as GCE.

GCE remains a major complication after SAH. Presently, assessment of GCE relies primarily on NCCT findings that are often difficult to identify in the setting of diffuse $\mathrm{SAH}$ and increased intracranial pressure. Given the influence of GCE on SAH morbidity and mortality, earlier detection of GCE may improve outcomes in $\mathrm{SAH}^{3}$

At present, there are no clinically established methods to assess the pathophysiologic mechanisms underlying GCE, such as microvascular dysfunction, inflammatory effects, and neurotoxic effects of blood. ${ }^{3}$ Given that these factors also play a role in BBB dysfunction, we have evaluated the utility of BBBP parameters in the assessment of GCE after SAH. However, these findings may be applicable to a wide spectrum of conditions where cerebral edema is known to occur, such as acute stroke, traumatic and anoxic brain injury, and CNS infections.

Our findings suggest that $\mathrm{K}_{\mathrm{ep}}$ decreases with increased $\mathrm{BBBP}^{14,15}$ and may represent the most useful parameter in GCE because it is independent of low blood flow conditions. This inverse relationship between $\mathrm{K}_{\mathrm{ep}}$ and $\mathrm{BBBP}$ could be attributed to the underlying pathophysiologic mechanisms, including time kinetics. Kinetic analysis of BBBP changes during focused sonography-induced BBB opening demonstrated initial decrease in $\mathrm{K}_{\mathrm{ep}}$ with subsequent recovery over time. ${ }^{16}$ Therefore, time kinetics are a crucial component in the assessment of the different BBBP parameters. In our study, we measured BBBP at a very early (initial) stage in patients with SAH who develop GCE demonstrating decreased $\mathrm{K}_{\mathrm{ep}}$. However, future work will show if $\mathrm{K}_{\mathrm{ep}}$ recovers on follow-up examinations in these patients. The exact timing of BBBP imaging during the course of the disease is an important factor in understanding $\mathrm{K}_{\mathrm{ep}}$ in these patients, as it relates to the timing of the BBB breakdown.

In contrast, $K^{\text {trans }}$ is dependent on blood flow and also decreased with increased BBBP in our study, representing a less reliable parameter for assessment of BBBP in GCE. Furthermore, our findings demonstrate significant differences in VE and VP in patients with GCE. The mathematical derivation of VE and VP is based on the fractions of volume of the EES and IVS, respectively. Fig 1 summarizes the relationships of these BBBP parameters under normal conditions and in the setting of GCE. In the setting of interstitial edema such as GCE, fluid from the IVS (represented by $\mathrm{VP}$ ) transgresses the BBB into the EES (represented by VE). In GCE, there is an increase in EES as reflected by increased VE and decreased $\mathrm{K}_{\mathrm{ep}}$, indicating increased BBBP. Furthermore, our findings also demonstrated significantly decreased $K^{\text {trans }}$ and $\mathrm{F}$ in patients with GCE. In the literature, CBF impairment recently has been reported in patients with GCE. ${ }^{12}$ Our data demonstrated decreased CBF in patients with GCE compared with patients without GCE and is thus concordant with previously published 
studies. ${ }^{12}$ Because the derivation of $K^{\text {trans }}$ is dependent on $F$, it is a less reliable parameter in flow-restricted conditions, such as seen in GCE. ${ }^{17}$ Given that $\mathrm{K}_{\mathrm{ep}}$ is independent of $\mathrm{F}$, it may represent a more useful parameter to assess BBBP in the context of GCE, particularly when flow impairment occurs. ${ }^{12}$ These findings further contribute to the development of an imaging biomarker of BBBP in flow-restricted conditions such as cerebral edema.

In this initial exploratory study, our sample was limited to patients with SAH who had an extended CTP technique performed. Future studies evaluating BBBP parameters in other patient populations such as stroke, hypoxic-ischemic injury, traumatic brain injury, neoplasm, and inflammation will be important to improving our understanding of BBBP in different clinical settings as well as to evaluate BBBP parameters as potential outcome measures in specific disease states.

Although there was a trend for increased PS in patients with GCE compared with patients without GCE, statistical significance was not achieved. However, these findings support further prospective work to elucidate the pathophysiologic processes leading to GCE after SAH.

\section{CONCLUSIONS}

Further study of BBBP is needed to improve diagnosis and monitoring of GCE. Validating a potential quantitative imaging biomarker may improve detection and management of GCE and may provide a quantitative end point for future clinical trials aimed at improving SAH outcomes.

Disclosures: Jana Ivanidze—RELATED: Grant: Radiological Society of North America Research and Education Foundation, ${ }^{*}$ Comments: Resident Research Grant 20142015. Ajay Gupta-RELATED: Grant: CTSC Pilot Award (NIH/NCATS Grant \# ULITR00457)*; UNRELATED: Grants/Grants Pending: Foundation of the American Society of Neuroradiology Scholar Award.* Jan Claassen-UNRELATED: Consultancy: Actelion, Sage, Comments: Advisory board for study planning. Pina C. Sanelli-RELATED: Grant: Brain Aneurysm Foundation Grant 2014-2015. *Money paid to the institution.

\section{REFERENCES}

1. Germanò A, d'Avella D, Imperatore C, et al. Time-course of bloodbrain barrier permeability changes after experimental subarachnoid haemorrhage. Acta Neurochir (Wien) 2000;142:575-80; discussion 580-81 CrossRef Medline

2. Kreiter KT, Copeland D, Bernardini GL, et al. Predictors of cognitive dysfunction after subarachnoid hemorrhage. Stroke 2002;33: 200-08 CrossRef Medline

3. Claassen J, Carhuapoma JR, Kreiter KT, et al. Global cerebral edema after subarachnoid hemorrhage: frequency, predictors, and impact on outcome. Stroke 2002;33:1225-32 CrossRef Medline

4. Sanelli PC, Anumula N, Johnson CE, et al. Evaluating CT perfusion using outcome measures of delayed cerebral ischemia in aneurysmal subarachnoid hemorrhage. AJNR Am J Neuroradiol 2013;34: 292-98 CrossRef Medline

5. Ivanidze J, Kesavabhotla K, Kallas ON, et al. Evaluating blood-brain barrier permeability in delayed cerebral infarction after aneurysmal subarachnoid hemorrhage. AJNR Am J Neuroradiol 2015;36: 850-54 CrossRef Medline

6. Gowland P, Mansfield P, Bullock P, et al. Dynamic studies of gadolinium uptake in brain tumors using inversion-recovery echo-planar imaging. Magn Reson Med 1992;26:241-58 CrossRef Medline

7. Tofts PS, Brix G, Buckley DL, et al. Estimating kinetic parameters from dynamic contrast-enhanced T(1)-weighted MRI of a diffusable tracer: standardized quantities and symbols. J Magn Reson Imaging 1999;10:223-32 Medline

8. Dankbaar JW, Hom J, Schneider T, et al. Dynamic perfusion CT assessment of the blood-brain barrier permeability: first pass versus delayed acquisition. AJNR Am J Neuroradiol 2008;29:1671-76 CrossRef Medline

9. Ozkul-Wermester O, Guegan-Massardier E, Triquenot A, et al. Increased blood-brain barrier permeability on perfusion computed tomography predicts hemorrhagic transformation in acute ischemic stroke. Eur Neurol 2014;72:45-53 CrossRef Medline

10. Hom J, Dankbaar JW, Schneider T, et al. Optimal duration of acquisition for dynamic perfusion CT assessment of blood-brain barrier permeability using the Patlak model. AJNR Am J Neuroradiol 2009; 30:1366-70 CrossRef Medline

11. Sanelli PC, Ugorec I, Johnson CE, et al. Using quantitative CT perfusion for evaluation of delayed cerebral ischemia following aneurysmal subarachnoid hemorrhage. AJNR Am J Neuroradiol 2011;32: 2047-53 CrossRef Medline

12. Baradaran H, Fodera V, Mir D, et al. Evaluating CT perfusion deficits in global cerebral edema after aneurysmal subarachnoid hemorrhage. AJNR Am J Neuroradiol 2015;36:1431-35 CrossRef Medline

13. St Lawrence KS, Lee TY. An adiabatic approximation to the tissue homogeneity model for water exchange in the brain: I. Theoretical derivation. J Cereb Blood Flow Metab 1998;18:1365-77 Medline

14. Bennink E, Riordan $\mathrm{AJ}$, Horsch $\mathrm{AD}$, et al. A fast nonlinear regression method for estimating permeability in CT perfusion imaging. J Cereb Blood Flow Metab 2013;33:1743-51 CrossRef Medline

15. Nance E, Timbie K, Miller GW, et al. Non-invasive delivery of stealth, brain-penetrating nanoparticles across the blood-brain barrier using MRI-guided focused ultrasound. J Control Release 2014;189:123-32 CrossRef Medline

16. Chai WY, Chu PC, Tsai MY, et al. Magnetic-resonance imaging for kinetic analysis of permeability changes during focused ultrasound-induced blood-brain barrier opening and brain drug delivery. J Control Release 2014;192:1-9 CrossRef Medline

17. Kety SS. The theory and applications of the exchange of inert gas at the lungs and tissues. Pharmacol Rev 1951;3:1-41 Medline 